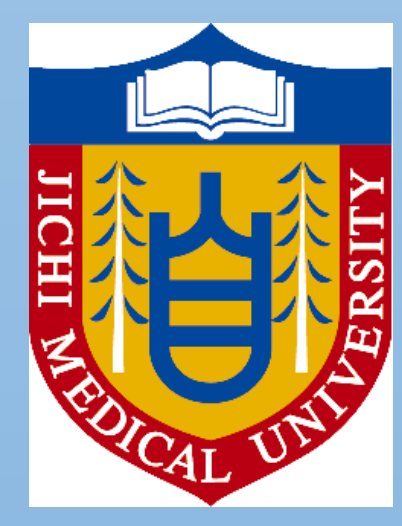

\title{
Does clinical experience affect the relationship between tracheal and bronchial cuff pressures \\ of double-lumen tubes?
}

Nozomu Hiraoka, Masaaki Satoh and Mamoru Takeuchi

Department of Anesthesiology and Critical care Medicine, Jichi Medical University, Japan

\section{Background and Goal of Study}

Appropriate management of the tracheal cuff pressure (TCP) and bronchial cuff pressure $(B C P)$ of a double lumen tube (DLT) is important during one lung ventilation.

However, the relationship between these pressures under general anesthesia arising from clinical experience is still unclear.

- We hypothesized that anesthesiologists' clinical experience does not affect these cuff pressure.

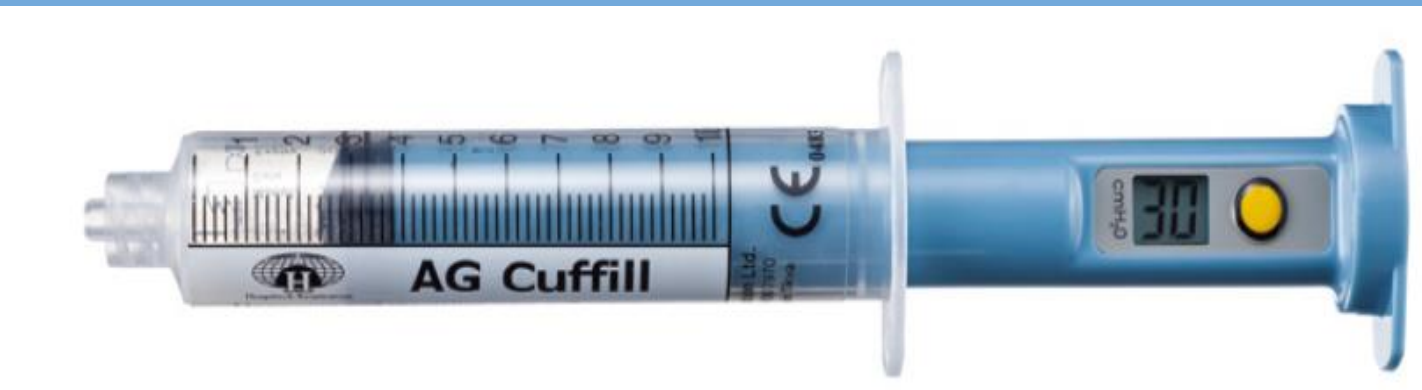

Courtesy by Covidien Japan Inc.

\section{Materials and Methods}

After approval of the Institutional Review Board (IRB), patients who had left-sided DLTs inserted were retrospectively investigated from anesthesia records between September 2016 and March 2017.

$>$ Anesthesiologists inflated cuffs without manometry. The BC was inflated via direct bronchoscopy through the tracheal lumen.

$>$ Anesthesiologists were divided into a fellow group and a non-fellow group based on clinical experience.

$>$ The cuff pressures were measured using a manometer at $5 \mathrm{~cm} \mathrm{H} 2 \mathrm{O}$ of positive end-expiratory pressure.

$>$ The patient characteristics, TCPs, the difference between TCPs and BCPs were statistically evaluated. A $p$ value $<0.05$ was considered significant. Statistical analysis was performed using JMP®12.0.1 software (SAS Institute Inc., Cary, NC, USA).

\section{Results and Discussion}

$>$ The number of anesthesiologists in the fellow and non-fellow groups were 9 and 5 , respectively.

$>$ The difference between the TCP and $\mathrm{BCP}$ (mean \pm standard deviation: SD) was $14.4 \pm 4.1 \mathrm{~cm} \mathrm{H} \mathrm{H}_{2} \mathrm{O}$ in the fellow group $(\mathrm{p}=0.003)$ and $7.4 \pm 8.7 \mathrm{~cm}$ $\mathrm{H}_{2} \mathrm{O}$ in the non-fellow group $(\mathrm{p}=0.41)$.

$>$ Wilcoxon singed-rank test showed that the BCP was significantly lower than the TCP in the fellow group, despite no significant differences between TCP and BCP in the non-fellow group.

$>$ More experienced anesthesiologists tended to avoid excessive BCP. It may be that clinical experience contributes to adjustment of cuff pressure.

\begin{tabular}{|l|c|c|c|}
\hline & $\begin{array}{c}\text { Fellow group } \\
(\mathbf{n}=17)\end{array}$ & $\begin{array}{c}\text { non-fellow group } \\
(\mathrm{n}=14)\end{array}$ & P value \\
\hline $\begin{array}{l}\text { Age (years), } \\
\text { mean } \pm \text { SD }\end{array}$ & $64.4 \pm 14.5$ & $64.5 \pm 13.0$ & $0.91^{\mathrm{a}}$ \\
\hline $\begin{array}{l}\text { Sex } \\
\text { (male:female) }\end{array}$ & $12: 5$ & $11: 3$ & $0.61^{\mathrm{b}}$ \\
\hline $\begin{array}{l}\text { Height }(\mathrm{cm}), \\
\text { mean } \pm \mathrm{SD}\end{array}$ & $162.8 \pm 6.0$ & $162.4 \pm 9.0$ & $0.88^{\mathrm{a}}$ \\
\hline $\begin{array}{l}\text { Weight }(\mathrm{kg}), \\
\text { mean } \pm \mathrm{SD}\end{array}$ & $61.5 \pm 8.4$ & $61.9 \pm 13.4$ & $0.93^{\mathrm{a}}$ \\
\hline $\begin{array}{l}\text { TCP }(\mathrm{cm} \mathrm{H} \mathrm{H}) \\
\text { mean } \pm \mathrm{SD}\end{array}$ & $40.6 \pm 18.9$ & $40.5 \pm 22.2$ & $0.99^{\mathrm{a}}$ \\
\hline $\begin{array}{l}\mathrm{BCP}(\mathrm{cm} \mathrm{H} \mathrm{H}) \\
\text { mean } \pm \mathrm{SD}\end{array}$ & $26.2 \pm 16.6$ & $33.1 \pm 21.4$ & $0.33^{\mathrm{a}}$ \\
\hline
\end{tabular}

\begin{tabular}{|c|c|c|}
\hline & $\begin{array}{l}\text { Fellow group } \\
(n=17)\end{array}$ & $\begin{array}{c}\text { non-fellow group } \\
(n=14)\end{array}$ \\
\hline $\begin{array}{l}\text { TCP-BCP }\left(\mathrm{cm} \mathrm{H}_{2} \mathrm{O}\right), \\
\text { mean } \pm \mathrm{SD}\end{array}$ & $14.4 \pm 4.1$ & $7.4 \pm 8.7$ \\
\hline$P$ Value ${ }^{c}$ & 0.003 & 0.41 \\
\hline
\end{tabular}

a Welch test, b Fisher's exact test, SD: standard deviation

c Wilcoxon singed-rank test, SD: standard deviation

\section{Conclusion}

Clinical experience affects the difference between TCP and BCP of DLT.

\section{References}

> Brodsky JB, Adkins MO, Gaba DM. Bronchial cuff pressures of double-lumen tubes. Anesth Analg. 1989; 69: 608-10. 